Addendum

\title{
Addendum: Montagnier, L.; Aïssa, J.; Capolupo, A.; Craddock, T.J.A.; Kurian, P.; Lavallee, C.; Polcari, A.; Romano, P.; Tedeschi, A.; Vitiello, G. Water Bridging Dynamics of Polymerase Chain Reaction in the Gauge Theory Paradigm of Quantum Fields. Water 2017, 9, 339
}

\author{
L. Montagnier ${ }^{1}$, J. Aïssa ${ }^{2}$, A. Capolupo ${ }^{3}$, T. J. A. Craddock ${ }^{4}$, P. Kurian ${ }^{5,6, *}$, C. Lavallee ${ }^{1}$, \\ A. Polcari ${ }^{7,8}$, P. Romano ${ }^{7,8}$, A. Tedeschi ${ }^{9}$ and G. Vitiello ${ }^{3, *}$ \\ 1 World Foundation for AIDS Research and Prevention, 75015 Paris, France; 1002175007mm@gmail.com \\ 2 Nanectis Biotechnologies, 78350 Jouy-en-Josas, France; 1002175007mm@gmail.com \\ 3 Dipartimento di Fisica “E.R. Caianiello", Universitá di Salerno and INFN, 84084 Fisciano (Salerno), Italy; \\ capolupo@sa.infn.it \\ 4 Departments of Psychology and Neuroscience, Computer Science, and Clinical Immunology, \\ and Clinical Systems Biology Group, Institute for Neuro-Immune Medicine, Nova Southeastern University, \\ Fort Lauderdale, FL 33314, USA; tcraddock@nova.edu \\ 5 National Human Genome Center and Department of Medicine, Howard University College of Medicine, \\ Washington, DC 20059, USA \\ 6 Computational Physics Laboratory, Howard University, Washington, DC 20059, USA \\ 7 Dipartimento di Scienze e Tecnologie, Universitá del Sannio, 82100 Benevento, Italy; \\ a.polcari@libero.it (A.P.); promano@unisannio.it (P.R.) \\ 8 SPIN-CNR, Universitá di Salerno, 84084 Fisciano (SA), Italy \\ 9 WHITE Holographic Bioresonance, Milano, Italy; gowhite@usa.net \\ * Correspondence: pkurian@howard.edu (P.K.); vitiello@sa.infn.it (G.V.)
}

Academic Editor: Astrid H. Paulitsch-Fuchs

Received: 16 June 2017; Accepted: 16 June 2017; Published: 17 June 2017

The authors wish to make the following addendum to their paper [1]:

After the publication of our paper, submitted on 11 February 2017, we read the paper "DNA's Chiral Spine of Hydration", by M. Luke McDermott, Heather Vanselous, Steven A. Corcelli, and Poul B. Petersen, published in ACS Central Science, doi:10.1021/acscentsci.7b00100, on 24 May 2017 [2], where the authors report their discovery of a chiral water superstructure surrounding DNA under ambient conditions. This is the first observation of a chiral spine of hydration templated by a biomolecule and has been obtained by use of chiral sum frequency generation (SFG) spectroscopy, a method analogous to circular dichroism measurements. In this specific case, water forms a robust chiral superstructure of the DNA helical structure. The authors report that, at room temperature and in $100 \mathrm{mM} \mathrm{NaCl}$ solution, they "indeed observe that DNA imprints its chirality on the surrounding water molecules, generating a chiral SFG water response. This confirms the existence of a DNA minor groove spine of hydration at room temperature and further shows that the chiral structure of biomolecules can be imprinted on the surrounding solvation structure". The biological relevance of the discovery is evident.

Here we observe that such a discovery is fully consistent with the predictions presented in our paper, namely that polymerase chain reaction (PCR) processes rest on the mediating influence of such hydration structures, templated by the DNA and Taq polymerase biomolecules. In fact, our conclusion is that, without organized water structures, no PCR processes can occur and no amplification of DNA can be obtained. The observation reported in [2] of the robustness of the DNA's chiral spine 
of hydration, and the fact that "a change in the hydration state can lead to dramatic changes to the DNA structure" [2] also confirm that such a water superstructure actually constitutes a detailed mold or "electromagnetic image", as we have called it in our paper, of the DNA (and, additionally, other biomolecules) generating molecular electric dipole interactions. This electromagnetic image imprinted in the water dipole field is what the polymerase enzyme recognizes in the DNA's water environment. These studies open the way toward novel biomolecular "imaging" technologies and new research disciplines, which have been incited by L. Montagnier's PCR experiments. The analysis in our paper, as well as in previous ones along similar research lines, is fully grounded in the quantum field theory description of the phenomenon and finds experimental support in this latest discovery of the existence of DNA's chiral spine of hydration.

Conflicts of Interest: The authors declare no conflict of interest.

\section{References}

1. Montagnier, L.; Aïssa, J.; Capolupo, A.; Craddock, T.J.A.; Kurian, P.; Lavallee, C.; Polcari, A.; Romano, P.; Tedeschi, A.; Vitiello, G. Water Bridging Dynamics of Polymerase Chain Reaction in the Gauge Theory Paradigm of Quantum Fields. Water 2017, 9, 339. [CrossRef]

2. McDermott, M.L.; Vanselous, H.; Corcelli, S.A.; Petersen, P.B. DNA's Chiral Spine of Hydration. ACS Cent. Sci. 2017. [CrossRef]

(C) 2017 by the authors. Licensee MDPI, Basel, Switzerland. This article is an open access article distributed under the terms and conditions of the Creative Commons Attribution (CC BY) license (http://creativecommons.org/licenses/by/4.0/). 